\title{
El gusano blanco de la papa. Premnotrypes vorax (Hustache) en Colombia. l.- Comportamiento de adultos en el campo
}

\section{Luis Valencia V.*}

\begin{abstract}
RESUMEN
Resultados de un experimento realizado en invernadero demostraron que la emergencia de los adultos del gusano blanco de la papa, (Premnotrypes vorax) (Hustache) es favorecida por el agua de riego. Con la ayuda de las trampas de caída se investigó la atracción de las plantas de papa a los adultos de esta especie bajo condiciones de campo. Se reporta también la fluctuación poblacional de los adultos a través de todo el ciclo del cultivo y la distribución de los adultos dentro de un campo de papas. El efecto de atracción ejercido por las trampas de caída cebadas con adultos de la misma especie, refuerzan el concepto de la probable existencia de una feromona de agrupamiento para esta especie.
\end{abstract}

Palabras Claves Adicionales: Gusano blanco, gorgojo de los Andes, Premnotrypes vorax, comportamiento del adulto, ecología, trampas de caída, riego.

Aceptado para publicación: Julio 18, 1989.

\footnotetext{
* Entomólogo, Región I Zona Andina, Centro Internacional de la Papa, CIP. Apartado Aéreo 92654 Bogotá, Colombia.
} 


\section{ABSTRACT}

\section{The Potato Andean Weevil, Premnotrypes vorax (Hustache) in Colombia. I.- Behavior of adults in the field}

An experiment carried out under screenhouse conditions showed that the emergence of the Andean weevil adult Premnotrypes vorax (Hustache) is favoured by the irrigation water. Using the pitfall traps under field conditions the attraction of adults by the potato plants was investigated. The population fluctuation of adults through the whole potato season, and the adult distribution into a potato field are also reported. The attraction of adults by pitfall traps baited with adults of the same species reinforce the concept of the existance of an aggregation pheromone for this species.

Additional Index Words: Gusano blanco, white grub, Andean weevil, Premnotrypes vorax, adult behavior, ecology, pitfall traps, irrigation.

El gusano blanco (GB) Premnotrypes vorax (Hustache) es la plaga más importante del cultivo de papa en las zonas altas de Colombia. El insecto en su forma adulta (Figura 1 a) se alimenta de las hojas de plantas de papa y de malezas. El daño más severo lo ocasionan las larvas (Figura 1 b), las cuales barrenan los tubérculos reduciendo el valor comercial de la cosecha. El principal problema que se tiene con esta plaga es que no existe una metodología de detección de adultos que permita cuantificar las poblaciones de campo. Se han efectuado varios intentos en este sentido, usando técnicas de detección por medio de zarandas (5), o con las trampas de caída $(6,8)$, las cuales proporcionan información relativa (13) acerca del tamaño de la población y la forma como varía ésta a través de todo el ciclo de cultivo. A pesar de que la información proporcionada por estos dispositivos es muy valiosa, lamentablemente estas técnicas consumen mucho tiempo para su implementación y mantenimiento, lo cual las hace poco prácticas para ser adoptadas por los agricultores.

Las trampas de caída se han usado hace algún tiempo para el estudio de artrópodos terrestres (4, 16, 9, 10, 11, 12 y 15). También han sido usadas para miembros del complejo "Gorgojo de los Andes"; hay reportes para $P$. suturicallus (2) y para $P$. vorax (6). Las trampas de caída son utilizadas por los adultos de GB como lugares de 
refugio, donde encuentran oscuridad y humedad relativa adecuada para permanecer durante el día. Pese a que la eficiencia de las trampas de caída es influenciada por aproximadamente 18 factores diferentes (1), la información que ellas proporcionan es muy importante siempre y cuando los datos se interpreten adecuadamente, poniendo especial atención a las potenciales fuentes de variación (13). Esta información debe ser complementada con otros parámetros relacionados con la biología del insecto, historia del campo en que se hace la investigación, factores que influyen en la emergencia de los adultos, etc.
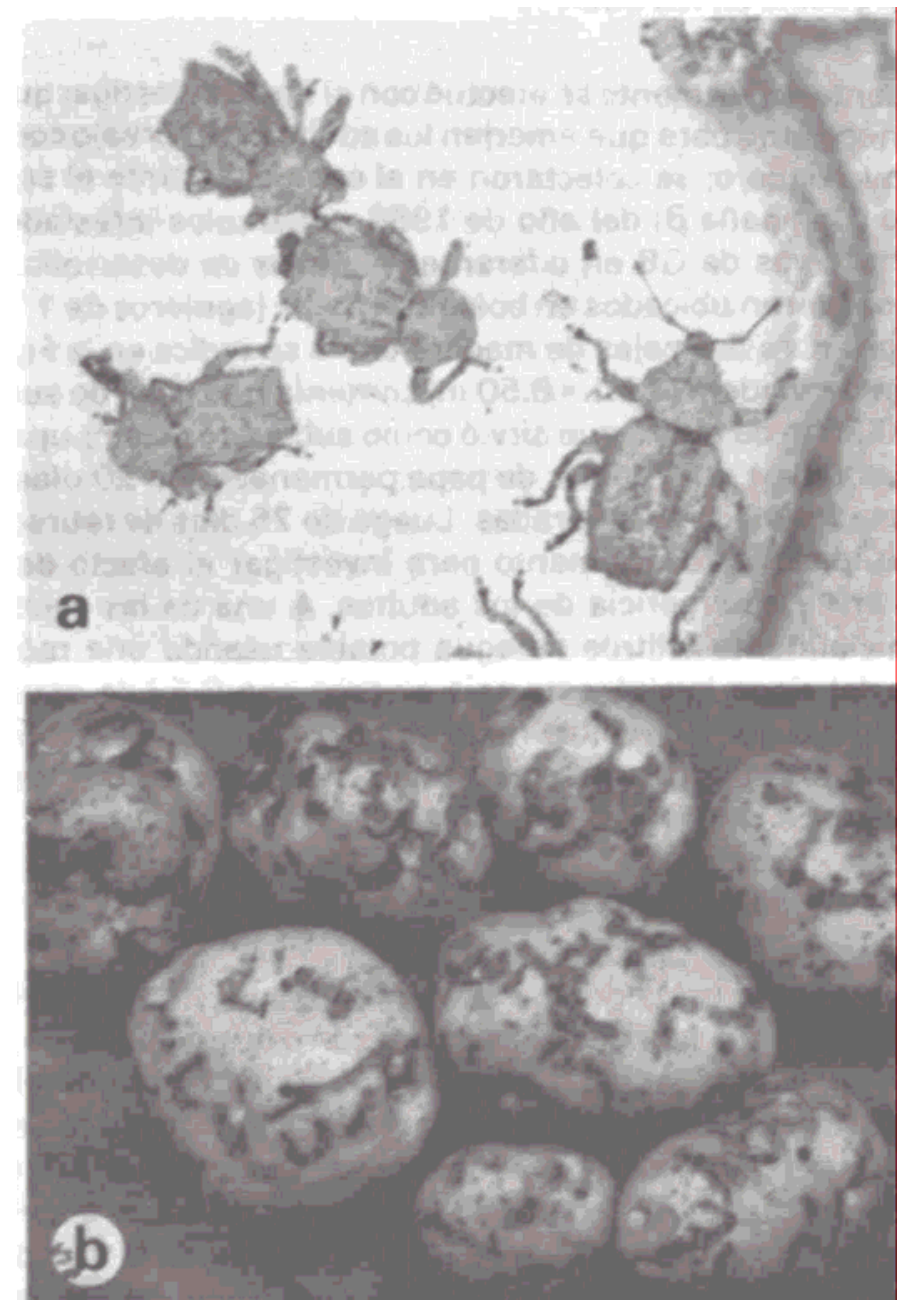

FIGURA 1.- a) Adultos de gusano blanco alimentándose en tajadas de papa.

b) Daño causado por las larvas de gusano blanco en tubérculos de papa. 
En 1985 se iniciaron investigaciones ecológicas acerca de adultos de GB que permitan enfrentar el problema de su control con una mejor base.

\section{MATERIALES Y MÉTODOS}

Los trabajos de invernadero y campo se efectuaron en el Centro Nacional de Investigaciones del Instituto Colombiano Agropecuario (ICA) en Tibaitatá (Bogotá), en el período comprendido entre 1985 y 1987.

\section{Emergencia de los adultos}

El presente experimento se efectuó con el fin de investigar qué estímulo es necesario para que emerjan los adultos de GB bajo condiciones de invernadero; se colectaron en el campo, durante el segundo semestre (Campaña B) del año de 1986 tubérculos infestados que contenían larvas de GB en diferentes estadios de desarrollo. Estos tubérculos fueron ubicados en bolsas de malla (agujeros de $1 \times 1 \mathrm{~cm}$.) y colgados sobre dos cajas de madera como se indica en la Figura 2. Cada caja de madera $(1 \times 1 \times 0.50 \mathrm{~m})$ contenía una capa de suelo vegetal de $0.30 \mathrm{~m}$ de altura que sirvió como substrato de empupamiento para las larvas. Las bolsas de papa permanecieron 20 días sobre cada caja para luego ser retiradas. Luego de 25 días de retiradas las bolsas se inició un experimento para investigar el efecto del agua de riego en la emergencia de los adultos. A una de las cajas se le aplicó inicialmente 2 litros de agua potable usando una regadera. Después del riego inicial, esta caja se regó con 0.5 I de agua cada 2 días. La caja que no recibió riego sirvió como testigo. La observación de adultos emergidos en las 2 cajas se hizo cada 2 días después de iniciados los riegos.

\section{Las trampas de caída}

Las trampas de caída que se usaron en los experimentos fueron construidas usando latas de aceite para carro de un cuarto de galón. Las dimensiones de las latas usadas fueron de $14 \mathrm{~cm}$. de altura y 10 $\mathrm{cm}$. de diámetro. Las latas fueron perforadas en su base con el fin de evitar se inundaran con el agua de lluvias. Las trampas fueron enterradas en el suelo tratando en lo posible que el borde superior se mantuviera al nivel del suelo circundante. La tapa de la trampa consistió en un círculo de madera prensada de $20 \mathrm{~cm}$. de diámetro, la cual reposaba en 3 piedras pequeñas sobre la lata enterrada, con el propósito de evitar el contacto directo entre la tapa y los bordes de la lata. La Figura 3 muestra un perfil de las trampas usadas. 


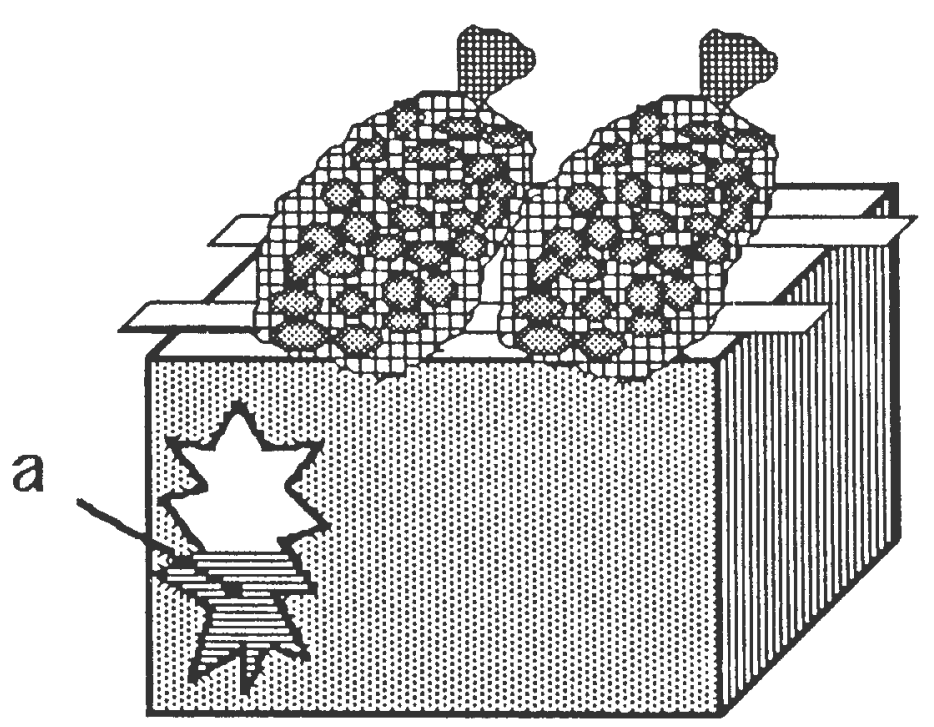

FIGURA 2. Dispositivo usado para investigar el efecto del agua de riego en la emergencia de los adultos de GB. a) Tierra vegetal usada como substrato de empupamiento de las larvas de GB.

\section{Experimentos realizados con las Trampas de Caída}

A) Influencia de la planta hospedera y de adultos vivos en la atracción de adultos de GB en las trampas de caída.

Para este experimento se incluyeron los siguientes tratamientos:

a) Trampas de caída en suelo descubierto.

b) Trampas de caída con 5 adultos de GB sin alimento dentro de la microjaula.

c) Trampas de caída con 5 adultos y con hojas de papa como alimento dentro de la microjaula.

d) Trampas de caída con 5 adultos de GB en la microjaula rodeadas por 5 plantas de papa.

e) Trampas de caída rodeadas por 5 plantas de papa solamente.

Las trampas fueron distribuidas al azar con un espaciamiento de aproximadamente $10 \mathrm{~m}$ entre trampas, en un campo descubierto que antes había mostrado niveles altos de infestación por GB. Cada uno de los tratamientos tuvo 5 repeticiones y el experimento duró 1 semana. Las plantas de papa fueron inicialmente sembradas en bolsas de plástico y luego, cuando alcanzaron aproximadamente 20 
cm de altura fueron transplantadas al campo.

B) Seguimiento de la población de campo de GB durante dos ciclos del cultivo.

Estos experimentos se realizaron en los semestres A y B de 1987. En un campo de aproximadamente 1 ha se instalaron 12 trampas de caída siguiendo el arreglo del tratamiento e) del experimento anterior. En este experimento los tubérculossemillas fueron inicialmente plantados en el invernadero en bolsas de plástico y cuando las plantas tuvieron aproximadamente $20 \mathrm{~cm}$ de altura, fueron transplantadas al campo. Se siguió este procedimiento con el fin de evitar fallas en la emergencia, lo cual podría influir en el efecto de atracción sobre adultos. Las trampas estuvieron distanciadas 10 m entre sí y las lecturas de adultos en las trampas se hicieron todos los días en 1987 y una vez por semana en 1987 B.

C) Observaciones de la distribución de los adultos dentro de un campo.

Para este experimento se siguió el mismo procedimiento citado en el experimento $B$. Se utilizaron 87 trampas de caída distribuidas en un campo de aproximadamente 1 ha; el experimento duró 15 días y las trampas fueron evaluadas todos los días.

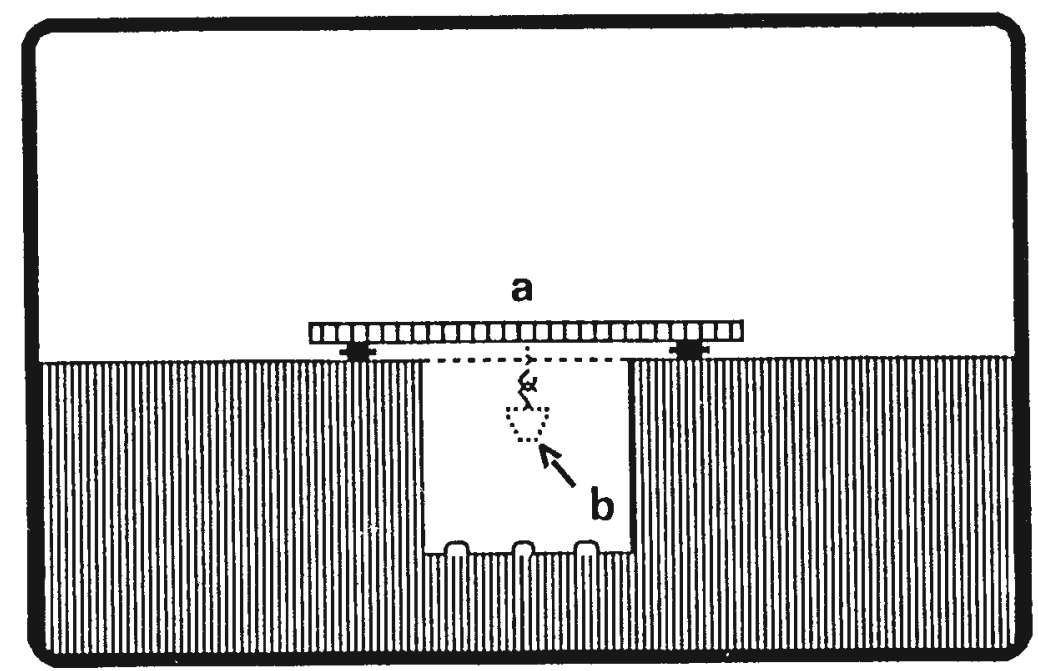

FIGURA 3. Diseño de la trampa de caída usada en los experimentos, a) Tapa de la trampa construida de madera triplex, b) Microjaula. 


\section{Atracción de adultos de GB por adultos de la misma especie}

Para la ejecución de este experimento se usaron trampas de caída con número variable de adultos encerrados en microjaulas. Se utilizaron densidades de 10, 25 y 50 adultos de ambos sexos (al azar) en las microjaulas, con 7 repeticiones por densidad. Cada trampa de caída estuvo rodeada de 5 plantas de papa del cultivar Monserrate. El experimento duró 1 semana y las evaluaciones se hicieron todos los días.

\section{RESULTADOS}

Se encontró que la emergencia de los adultos de GB está fuertemente influenciada por el agua de riego. Los resultados se muestran en la Figura 4. Se observó que a los 2 días de iniciado el experimento comenzó la emergencia de adultos en la caja que recibió riego, mientras que en la caja sin riego no emergió ningún adulto durante todo el período de observación. Posteriormente, también se aplicó riego a la caja que no había recibido y se recuperó en los días siguientes una cantidad de adultos parecida a la obtenida en la caja con riego del experimento, lo anterior demuestra que los adultos aún estaban vivos esperando el estímulo del agua.

Los resultados de la influencia de las plantas de papa en la atracción de adultos de GB hacia las trampas de caída, se muestran en la Figura 5. Las trampas de caída que estuvieron acompañadas de plantas de papa, capturaron el mayor número de adultos de GB, mientras que las trampas sin plantas capturaron un número muy reducido de adultos.

Resultados del seguimiento de las poblaciones de campo de GB a través de todo el ciclo del cultivo se muestran en las Figuras 6 y 7 . Se observó que las capturas al inicio del cultivo fueron altas (Figura 6), para luego ir descendiendo regularmente con la edad del cultivo. Cuando los datos del año 1986 (lecturas diarias) se arreglaron a evaluaciones interdiarias, se observó que en los primeros 20 días después del transplante, se produjo una captura más consistente (valores menores de las D. E. de las muestras) para luego iniciarse el descenso de las capturas (Figura 7).

Los resultados sobre la distribución de adultos dentro de un campo se muestran en la Figura 8. Se observó que durante varios días consecutivos algunas trampas dentro del campo capturaban una mayor proporción de adultos. Los resultados de la Figura 8, 
Número de adultos emergidos
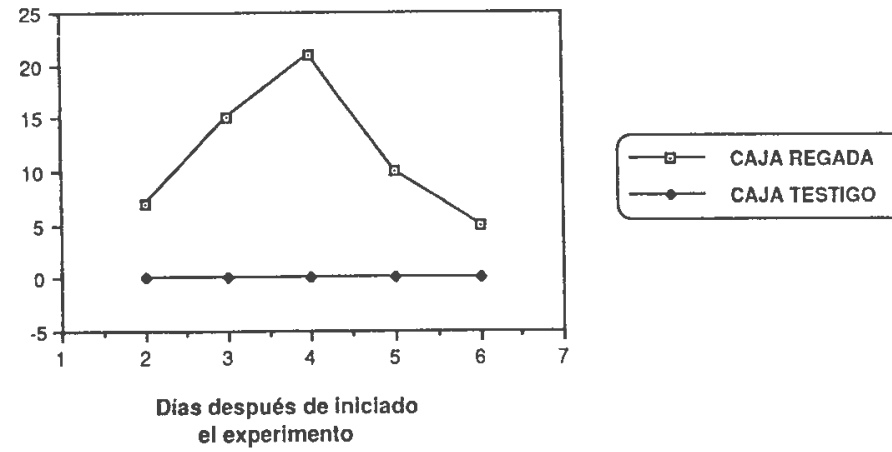

FIGURA 4. Efecto del agua de riego en la emergencia de los adultos del gusano blanco.

\section{$\overline{\mathrm{X}}$ de adultos/trampa/semana}

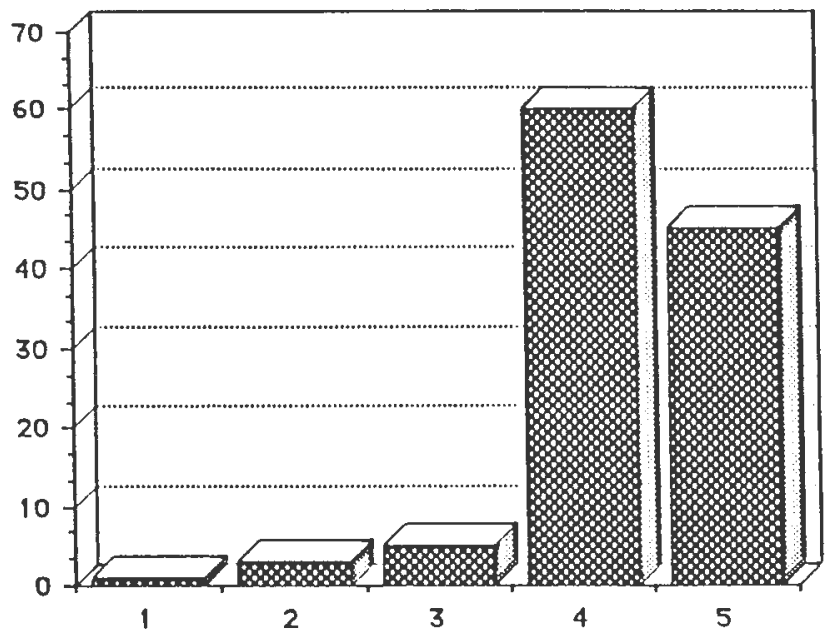

\section{Tratamientos}

FIGURA 5. Captura de adultos de gusano blanco con los tratamientos siguientes: 1) Trampa de caída en suelo descubierto. 2) Trampa de caída con 5 adultos sin alimento dentro de la microjaula, 3) Trampa de caída con 5 adultos con hojas de papa como alimento dentro de la microjaula, 4) Trampa de caída con 5 adultos en la microjaula rodeada de 5 plantas de papa, 5) Trampa de caída rodeada de 5 plantas de papa solamente. 
No. de adultos/trampa

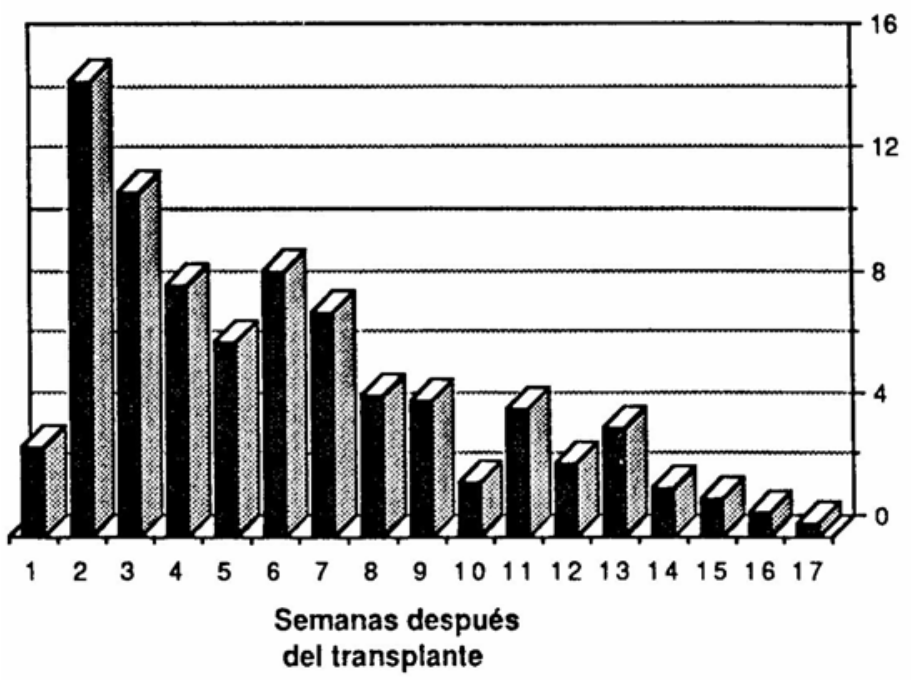

FIGURA 6. Fluctuación de la población de adultos de gusano blanco a través de todo el ciclo del cultivo.

\section{$\bar{X}$ de adultos $(\log ) /$ trampa}

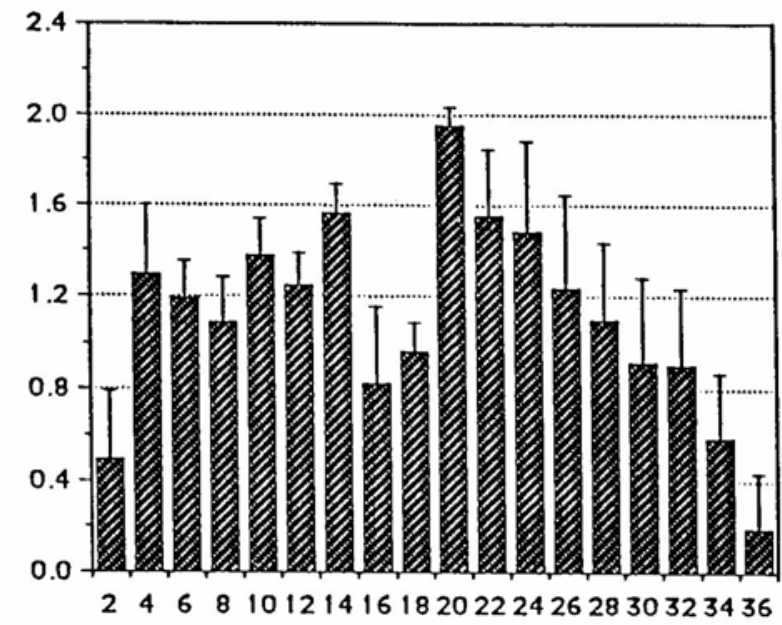

Días después del transplante

FIGURA 7. Fluctuación de la población de adultos (lecturas interdiarias) con promedios transformados (Log) más la D. E. que demuestra la consistencia de las capturas durante los primeros 20 días después del transplante. 


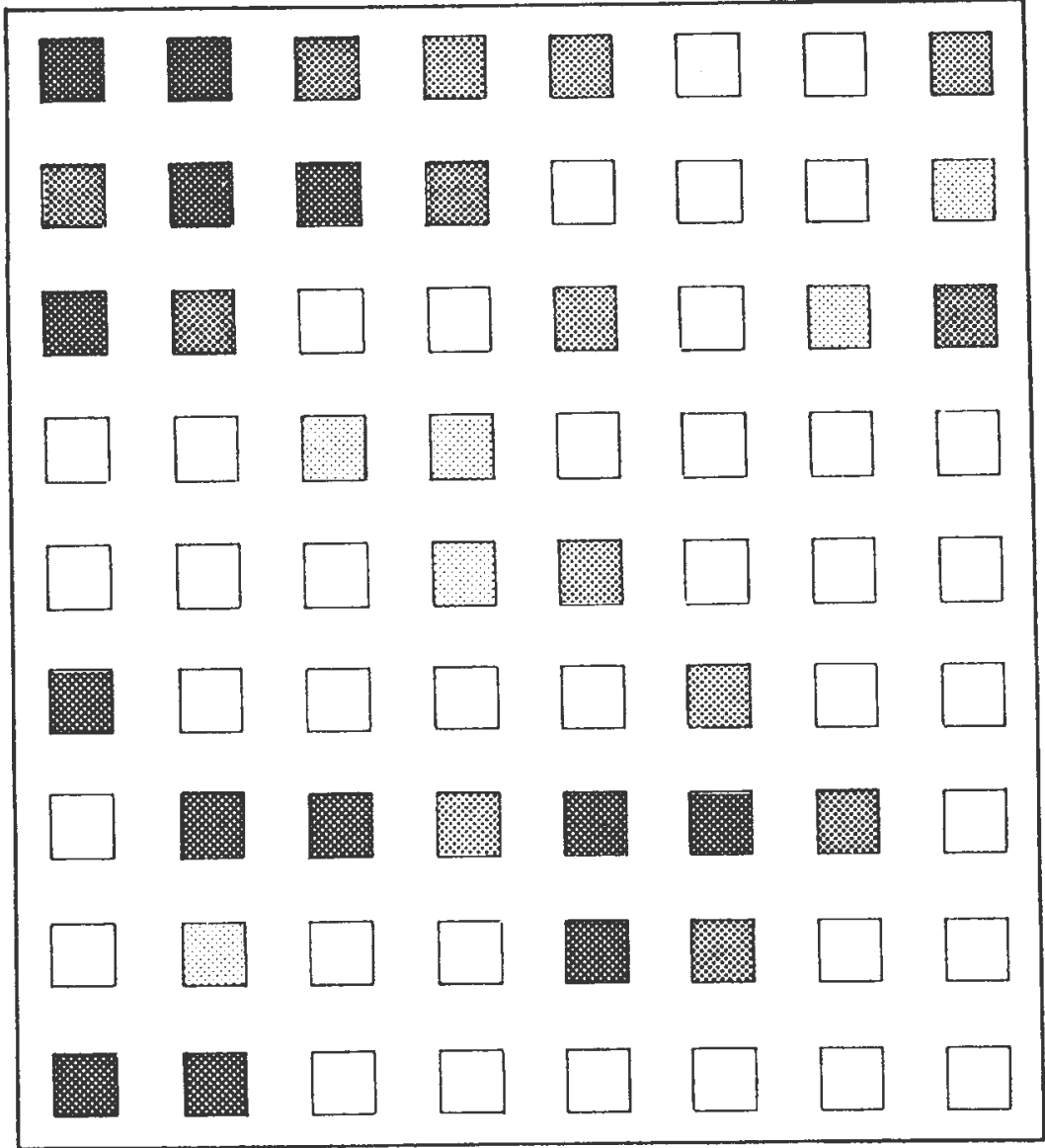

FIGURA 8. Distribución de adultos de Gusano Blanco, $P$. vorax en un campo de 1 ha determinado por las trampas de caída.
$\square$
0-5 adultos/trampa

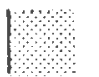
6-10 adultos/trampa
11-15 adultos/trampa
16-20 adultos/trampa

más de 21 adultos/trampa 
pertenecen a las capturas de un día, y se puede observar cierto efecto de agregación de los adultos en algunos sectores del campo.

Los resultados sobre la atracción de adultos de GB por adultos de la misma especie se muestran en la Tabla 1. Se observa claramente que a medida que se incrementa el número de adultos en las microjaulas, se incrementa también el número de adultos capturados por las trampas.

TABLA 1. Atracción diaria de adultos de gusano blanco por trampas de caída cebadas con tres densidades de adultos mantenidos en microjaulas.

* Los promedios seguidos por letras distintas, difieren significativamente; Prueba de Duncan $(p=0.01)$.

\section{DISCUSIÓN}

La influencia de la humedad en el suelo (del agua de lluvias) en la emergencia de los adultos del GB y de otras especies estrechamente relacionadas que pertenecen al grupo "Gorgojos de los Andes", ha sido sugerida por varios autores (14, 3, 8 y 17). Sin embargo, ésta es la primera vez que se reportan resultados experimentales que demuestran la gran influencia del agua en la emergencia de los adultos del GB de la especie $P$. vorax. Con esta información se pueden mejorar las estrategias de control para esta especie.

Resultados del uso de las trampas de caída acompañadas con plantas de papa en el arreglo e) usado en el experimento $3 \mathrm{~A}$, demuestran claramente el efecto atrayente de las plantas de papa para los adultos del GB. Estos resultados concuerdan con lo reportado en la literatura (8), y podrían utilizarse en estudios de detección de la plaga en campos nuevos que se piensan dedicar para el cultivo de papa. Parece que las trampas de caída solas no constituyen un buen dispositivo para la detección de los adultos, excepto cuando la población de adultos en el campo es muy grande, tal como ha sido demostrado (8). La fluctuación de la población de 
adultos de GB a través de todo el ciclo del cultivo concuerda bien con los resultados reportados (8), las capturas son altas al comienzo del cultivo, para luego descender en forma regular. Debido a que tanto los resultados que han sido reportados previamente (8) y a los de este artículo, fueron obtenidos con las trampas de caída, es importante considerar las ventajas y desventajas de esta metodología. Greenslade (11), quien usó trampas de caída para hormigas en Australia reportó el efecto de extracción (digging in efect) de adultos de insectos de una población, y demostró que las capturas son altas al comienzo para luego disminuir lentamente.

Si se considera que el GB tiene una generación de adultos cada 3.21 meses bajo las condiciones de Tibaitatá (Zenner de Polanía y Posada, 1968), esto significa que tanto Calvache (8) como el autor iniciaron los muestreos con una población conocida, más o menos estable, que sólo pudo ser influenciada por defectos de la misma técnica de muestreo y por adultos migrantes que vienen de áreas retiradas al campo en que se está experimentando. Al iniciar los muestreos con una población estable, las trampas empiezan un proceso de extracción de miembros de la población de adultos que lógicamente tenderá a disminuir con el tiempo. Los resultados obtenidos con las trampas de caída han sido considerados experimentalmente por Adis (1). El reconoce que no todos los artrópodos que son activos en la superficie del suelo caen en las trampas, la captura está fuertemente influenciada por diferencias en el comportamiento de los artrópodos. Insectos como los adultos de GB, en los que se sospecha de la existencia de una feromona de agrupamiento (8), caen con mayor profusión en las trampas de caída de acuerdo con lo establecido por Adis (1).

Los adultos de $P$. vorax tienden a buscar las zonas más húmedas dentro de un campo de cultivo; este comportamiento también ha sido reportado para $P$. suturicallus en el Perú (3). Resultados experimentales que se presentan en este artículo refuerzan el concepto de Calvache (8), en el sentido de la probable existencia de una feromona de agrupamiento. No obstante, los resultados presentados aquí no concuerdan con los de Calvache (8), quien utilizó adultos vírgenes de ambos sexos como atrayente. La diferencia podría atribuirse al número de individuos usados en cada microjaula, ya que el máximo número de adultos usados por él fue de 12 individuos, mientras que en el presente trabajo se utilizaron densidades de hasta 50 individuos por microjaula.

En la actualidad se conoce bien la ecología de los adultos de GB y de las larvas (ver trabajo por Valencia en este mismo volumen), lo que permite iniciar investigaciones acerca del control de esta plaga. 


\section{Agradecimientos}

El autor agradece al personal de apoyo de la Oficina Regional del CIP en Bogotá (Región I) y al ICA por las facilidades prestadas para la realización de esta investigación.

\section{REFERENCIAS BIBLIOGRÁFICAS}

1. Adis, J. 1979. Problems of interpreting arthropod sampling with pitfall traps. Zool. Anz. Jena 202: 177-184.

2. Alcalá, J. G. 1975. Memoria Anual. Estación Experimental Agraria de la Sierra Central. Huancayo. Ministerio de Alimentación. CRIA I. La Molina.

3. Alcázar, J. G. 1976. Biología y comportamiento del "Gorgojo de los Andes" Premnotrypes suturicallus Kuschel (Coleóptera: Curculionidae). Tesis para optar título de Ingeniero Agrónomo. Universidad Nacional del Centro del Perú. Huancayo. $80 \mathrm{p}$.

4. Barber, H. 1931. Traps for cave-inhabiting insects. J. Elisha Mitchell Sci. Soc. 46: $259-266$

5. Calvache, H. 1979. Método de "zarandas" para detectar la presencia de adultos de gusano blanco de la papa, Premnotrypes vorax (Hustache) en el suelo. Revista Colombiana de Entomología 5 (1 -2): 31 -35.

6. Calvache, H. 1984. Detección de adultos de gusano blanco de la papa, Premnotrypes vorax (Hustache) en el campo. XII Reunión de ALAP. PaipaBoyacá (Colombia): 585-593.

7. Calvache, H. 1986. Aspectos biológicos y ecológicos del gusano blanco de la papa Premnotrypes vorax (Hustache). p.p. 18-24. En: Valencia, L. (Edit.), Memorias del Curso sobre Control Integrado de Plagas de Papa. CIP-ICA, Colombia.

8. Calvache, H. 1987. Captura de adultos de Premnotrypes vorax (Hustache) con adultos de la misma especie como atrayente. Revista Colombiana de Entomología 11 (2), (vol. correspondiente a 1985): 9-14.

9. Fichter, E. 1941. Apparatus for the comparison of soil surface arthropod populations. Ecology 22: 338-339.

10. Greenslade, P. J. M. 1964. Pitfall trapping as a method for studying populations of Carabidae (Coleoptera). J. Anim. Ecol. 33: 301 -310.

11. Greenslade, P. J. M. 1973. Sampling ants with pitfall traps: Digging-in effects. Insectes Sociaux, Paris, 20 (4): 343-353.

12. Holopainen, J. K.; Varis, A. L. 1986. Effects of a mechanical barrier and formalin preservative on pitfall catches of carabid beetles (Coleóptera, Carabidae) in arable fields. J. Appl. Ent. 102: 440-445. 
13. Southwood, T. R. E. 1978. Ecological Methods with particular reference to the study of insect populations. "A Halsted Press Book", London, Chapman and Hall. 524 p.

14. Valencia, L. 1975. Problemas entomológicos de la papa. Centro Internacional de la Papa. Mimeo. 27 p.

15. Wallin, H. 1985. Spatial and temporal distribution of some abundant carabid beetles (Coleoptera: Carabidae) in aereal fields and adjacent habitats. Pedobiologia 28: 19-34.

16. Walsh, G. B. 1933. Studies in the British necrophagous Coleoptera. II. The attractive powers of various natural baits. Ent. Mom. Mag. 69: 28-32.

17. Yabar, E. 1988. Integración de prácticas culturales para el control del gorgojo de los Andes (Premnotrypes ssp.). Revista Latinoamericana de la Papa 1 (1): 120-131.

18. Zenner de Polanía, I.; Posada. L. 1968. Generalidades sobre el gusano blanco de la papa, Premnotrypes vorax (Hustache). Bogotá, Colombia. Agricultura Tropical 24(1): 33-40. 